\title{
Introduction to Neutrosophic BCI/BCK-Algebras
}

\author{
A. A. A. Agboola ${ }^{1}$ and B. Davvaz \\ ${ }^{1}$ Department of Mathematics, Federal University of Agriculture, Abeokuta, Nigeria \\ ${ }^{2}$ Department of Mathematics, Yazd University, Yazd, Iran
}

Correspondence should be addressed to A. A. A. Agboola; aaaola2003@yahoo.com

Received 4 February 2015; Accepted 23 February 2015

Academic Editor: Sergejs Solovjovs

Copyright (c) 2015 A. A. A. Agboola and B. Davvaz. This is an open access article distributed under the Creative Commons Attribution License, which permits unrestricted use, distribution, and reproduction in any medium, provided the original work is properly cited.

We introduce the concept of neutrosophic BCI/BCK-algebras. Elementary properties of neutrosophic BCI/BCK algebras are presented.

\section{Introduction}

Logic algebras are the algebraic foundation of reasoning mechanism in many fields such as computer sciences, information sciences, cybernetics, and artificial intelligence. In 1966, Imai and Iséki $[1,2]$ introduced the notions, called $\mathrm{BCK}$-algebras and BCI-algebras. These notions are originated from two different ways: one of them is based on set theory; another is from classical and nonclassical propositional calculi. As is well known, there is a close relationship between the notions of the set difference in set theory and the implication functor in logical systems. Since then many researchers worked in this area and lots of literatures had been produced about the theory of BCK/BCI-algebra. On the theory of $\mathrm{BCK} / \mathrm{BCI}$-algebras, for example, see [2-6]. It is known that the class of BCK-algebras is a proper subclass of the class of BCI-algebras. MV-algebras were introduced by Chang in [7], in order to show that Lukasiewicz logic is complete with respect to evaluations of propositional variables in the real unit interval $[0,1]$. It is well known that the class of MValgebras is a proper subclass of the class of BCK- algebras.

By a BCI-algebra we mean an algebra $(X, *, 0)$ of type $(2,0)$ satisfying the following axioms, for all $x, y, z \in X$ :

(1) $((x * y) *(x * z)) *(z * y)=0$,

(2) $(x *(x * y)) * y=0$,

(3) $x * x=0$,

(4) $x * y=0$ and $y * x=0$ imply $x=y$.
We can define a partial ordering $\leq$ by $x \leq y$ if and only if $x * y=0$.

If a BCI-algebra $X$ satisfies $0 * x=0$ for all $x \in X$, then we say that $X$ is a BCK-algebra. Any BCK-algebra $X$ satisfies the following axioms for all $x, y, z \in X$ :

(1) $(x * y) * z=(x * z) * y$,

(2) $((x * z) *(y * z)) *(x * y)=0$,

(3) $x * 0=x$,

(4) $x * y=0 \Rightarrow(x * z) *(y * z)=0,(z * y) *(z * x)=0$.

Let $(X, *, 0)$ be a BCK-algebra. Consider the following:

(1) $X$ is said to be commutative if for all $x, y \in X$ we have $x *(x * y)=y *(y * x)$

(2) $X$ is said to be implicative if for all $x, y \in X$, we have $x=x *(y * x)$.

In 1995, Smarandache introduced the concept of neutrosophic logic as an extension of fuzzy logic; see [8-10]. In 2006, Kandasamy and Smarandache introduced the concept of neutrosophic algebraic structures; see $[11,12]$. Since then, several researchers have studied the concepts and a great deal of literature has been produced. Agboola et al. in [13-17] continued the study of some types of neutrosophic algebraic structures.

Let $X$ be a nonempty set. A set $X(I)=\langle X, I\rangle$ generated by $X$ and $I$ is called a neutrosophic set. The elements of $X(I)$ are of the form $(x, y I)$, where $x$ and $y$ are elements of $X$. 
In the present paper, we introduce the concept of neutrosophic BCI/BCK-algebras. Elementary properties of neutrosophic BCI/BCK-algebras are presented.

\section{Main Results}

Definition 1. Let $(X, *, 0)$ be any $\mathrm{BCI} / \mathrm{BCK}$-algebra and let $X(I)=\langle X, I\rangle$ be a set generated by $X$ and $I$. The triple $(X(I), *,(0,0))$ is called a neutrosophic BCI/BCK-algebra. If $(a, b I)$ and $(c, d I)$ are any two elements of $X(I)$ with $a, b, c, d \in$ $X$, we define

$$
(a, b I) *(c, d I)=(a * c,(a * d \wedge b * c \wedge b * d) I) .
$$

An element $x \in X$ is represented by $(x, 0) \in X(I)$ and $(0,0)$ represents the constant element in $X(I)$. For all $(x, 0),(y, 0) \in$ $X$, we define

$$
(x, 0) *(y, 0)=(x * y, 0)=(x \wedge \neg y, 0),
$$

where $\neg y$ is the negation of $y$ in $X$.

Example 2. Let $(X(I),+)$ be any commutative neutrosophic group. For all $(a, b I),(c, d I) \in X(I)$ define

$$
(a, b I) *(c, d I)=(a, b I)-(c, d I)=(a-c,(b-d) I) .
$$

Then $(X(I), *,(0,0))$ is a neutrosophic BCI-algebra.

Example 3. Let $X(I)$ be a neutrosophic set and let $A(I)$ and $B(I)$ be any two nonempty subsets of $X(I)$. Define

$$
A(I) * B(I)=A(I)-B(I)=A(I) \cap B^{\prime}(I) .
$$

Then $(X(I), *, \emptyset)$ is a neutrosophic BCK-algebra.

Theorem 4. Every neutrosophic BCK-algebra $(X(I), *,(0,0))$ is a neutrosophic BCI-algebra.

Proof. It is straightforward.

Theorem 5. Every neutrosophic BCK-algebra $(X(I), *,(0,0))$ is a BCK-algebra and not the converse.

Proof. Suppose that $(X(I), *,(0,0))$ is a neutrosophic BCKalgebra. Let $x=(a, b I), y=(c, d I)$, and $z=(e, f I)$ be arbitrary elements of $X(I)$. Then we have the following.

(1) We have

$$
\begin{aligned}
& ((x * y) *(x * z)) *(z * y) \\
& \quad=(((a, b I) *(c, d I)) *((a, b I) *(e, f I))) \\
& \quad *((e, f I) *(c, d I)) \\
& \quad \equiv[(r, s I) *(p, q I)] *(u, v I),
\end{aligned}
$$

where

$$
\begin{aligned}
(r, s I) & =(a * c,(a * d \wedge b * c \wedge b * d) I) \\
& =(a \wedge \neg c,(a \wedge \neg d \wedge b \wedge \neg c) I), \\
(p, q I) & =(a * e,(a * f \wedge b * e \wedge b * f) I) \\
& =(a \wedge \neg e,(a \wedge \neg f \wedge b \wedge \neg e) I), \\
(u, v I) & =(e * c,(e * d \wedge f * c \wedge f * d) I) \\
& =(e \wedge \neg c,(e \wedge \neg d \wedge f \wedge \neg c) I) .
\end{aligned}
$$

Hence,

$$
\begin{aligned}
(r, s I) *(p, q I) & =(r * p,(r * q \wedge s * p \wedge s * q) I) \\
& =(r \wedge \neg p,(r \wedge \neg q \wedge s \wedge \neg p) I) \\
& \equiv(m, k I), \\
(m, k I) *(u, v I) & =(m * u,(m * v \wedge k * u \wedge k * v) I) \\
& =(m \wedge \neg u,(m \wedge \neg v \wedge k \wedge \neg u) I) \\
& \equiv(g, h I) .
\end{aligned}
$$

Now, we obtain

$$
\begin{aligned}
g & =m \wedge \neg u=r \wedge \neg p \wedge \neg \mathcal{u} \\
& =(a \wedge \neg c \wedge e)(\neg e \vee c)=0 .
\end{aligned}
$$

Also, we have

$$
\begin{aligned}
h & =m \wedge \neg v \wedge k \wedge \neg u \\
& =r \wedge \neg p \wedge \neg q \wedge s \wedge \neg v \wedge \neg u \\
& =a \wedge \neg c \wedge \neg d \wedge b \wedge \neg p \wedge \neg q \wedge \neg v \wedge \neg u \\
& =a \wedge \neg c \wedge \neg d \wedge b \wedge(\neg a \vee e) \wedge(\neg e \vee c) \wedge \neg q \wedge \neg v \\
& =a \wedge \neg c \wedge \neg d \wedge b \wedge e \wedge(\neg e \vee c) \wedge \neg q \wedge \neg v \\
& =0 .
\end{aligned}
$$

This shows that $(g, h I)=(0,0)$ and, consequently, $((x * y) *$ $(x * z)) *(z * y)=0$.

(2) We have

$$
\begin{aligned}
(x & *(x * y)) * y \\
& =((a, b I) *((a, b I) *(c, d I))) *(c, d I) \\
& =((a, b I) *(a * c,(a * d \wedge b * c \wedge b * d) I)) *(c, d I) \\
& =((a, b I) *(r, s I)) *(c, d I),
\end{aligned}
$$

where

$$
\begin{aligned}
(r, s I) & =(a, b I) *(c, d I) \\
& =(a * c,(a * d \wedge b * c \wedge b * d) I) \\
& =(a \wedge \neg c,(a \wedge \neg d \wedge b \wedge \neg c) I) .
\end{aligned}
$$


Then,

$$
\begin{aligned}
(a, b I) *(r, s I) & =(a * r,(a * s \wedge b * r \wedge b * s) I) \\
& =(a \wedge \neg r,(a \wedge \neg s \wedge b \wedge \neg r) I) \\
& \equiv(u, v I) .
\end{aligned}
$$

Therefore, we obtain

$$
\begin{aligned}
(u, v I) *(c, d I) & =(u * c,(u * d \wedge v * c \wedge v * d) I) \\
& =(u \wedge \neg c, u \wedge \neg d \wedge v \wedge \neg c) \\
& \equiv(p, q I),
\end{aligned}
$$

where

$$
\begin{aligned}
p & =u \wedge \neg c=a \wedge \neg r \wedge \neg c \\
& =a \wedge(\neg a \vee c) \wedge \neg c=a \wedge c \wedge \neg c=0, \\
q & =u \wedge \neg d \wedge v \wedge \neg c=a \wedge \neg r \wedge \neg d \wedge v \wedge \neg c \\
& =a \wedge(\neg a \vee c) \wedge \neg d \wedge v \wedge \neg c \\
& =a \wedge c \wedge \neg d \wedge v \wedge \neg c=0 .
\end{aligned}
$$

Since $(p, q I)=(0,0)$, it follows that $(x *(x * y)) * y=0$.

(3) We have

$$
\begin{aligned}
x * x & =(a, b I) *(a, b I) \\
& =(a * a,(a * b \wedge b * a \wedge b * b) I) \\
& =(a \wedge \neg a,(a \wedge \neg b \wedge b \wedge \neg a \wedge b \wedge \neg b) I) \\
& =(0,0) .
\end{aligned}
$$

(4) Suppose that $x * y=0$ and $y * x=0$. Then $(a, b I) *$ $(c, d I)=(0,0)$ and $(c, d I) *(a, b I)=(0,0)$ from which we obtain $(a * c,(a * d \wedge b * c \wedge b * d) I)=(0,0)$ and $(c * a,(c * b \wedge d * a \wedge d * b) I)=(0,0)$. These imply that $(a \wedge \neg c,(a \wedge \neg d \wedge b \neg c) I)=(0,0)$ and $(c \wedge \neg a,(c \wedge \neg b \wedge$ $d \neg a) I)=(0,0)$ and therefore, $a \wedge \neg c=0, a \wedge \neg d \wedge b \neg c=$ $0, c \wedge \neg a=0$, and $c \wedge \neg b \wedge d \neg a=0$ from which we obtain $a=c$ and $b=d$. Hence, $(a, b I)=(c, d I)$; that is, $x=y$.

(5) We have

$$
\begin{aligned}
0 * x & =(0,0) *(a, b I)=(0 * a,(0 * b \wedge 0 * a) I) \\
& =(0,(0 \wedge 0) I)=(0,0) .
\end{aligned}
$$

Items $(1)-(5)$ show that $(X(I), *,(0,0))$ is a BCK-algebra.

Lemma 6. Let $(X(I), *,(0,0))$ be a neutrosophic $B C K-$ algebra. Then $(a, b I) *(0,0)=(a, b I)$, if and only if $a=b$.

Proof. Suppose that $(a, b I) *(0,0)=(a, b I)$. Then $(a * 0,(a *$ $0 \wedge b * 0) I)=(a, b I)$ which implies that $(a,(a \wedge b) I)=(a, b I)$ from which we obtain $a=b$. The converse is obvious.
Lemma 7. Let $(X(I), *,(0,0))$ be a neutrosophic BCI-algebra. Then for all $(a, b I),(c, d I) \in X(I)$,

$$
\begin{aligned}
& \text { (1) }(0,0) *((a, b I) *(c, d I))=((0,0) *(a, b I)) *((0,0) * \\
& \quad(c, d I)) ; \\
& \text { (2) }(0,0) *((0,0) *((a, b I) *(c, d I)))=(0,0) *((a, b I) * \\
& \quad(c, d I)) .
\end{aligned}
$$

Theorem 8. Let $(X(I), *,(0,0))$ be a neutrosophic BCKalgebra. Then for all $(a, b I),(c, d I),(e, f I) \in X(I)$,

(1) $(a, b I) *(c, d I)=(0,0)$ implies that $((a, b I) *(e, f I)) *$ $((c, d I) *(e, f I))=(0,0)$ and $((e, f I) *(c, d I)) *$ $((e, f I) *(a, b I))=(0,0)$;

(2) $((a, b I) *(c, d I)) *(e, f I)=((a, b I) *(e, f I)) *(c, d I)$;

(3) $((a, b I) *(e, f I)) *((c, d I) *(e, f I)) *((a, b I) *(c, d I))=$ $(0,0)$.

Proof. (1) Suppose that $(a, b I) *(c, d I)=(0,0)$. Then $(a *$ $c,(a * d \wedge b * c \wedge b * d) I)=(0,0)$ from which we obtain

$$
a \wedge \neg c=0, \quad a \wedge \neg d \wedge b \wedge \neg c=0 .
$$

Now,

$$
\begin{aligned}
(x, y I) & =(a, b I) *(e, f I) \\
& =(a \wedge \neg e,(a \wedge \neg f \wedge b \wedge \neg e) I), \\
(p, q I) & =(c, d I) *(e, f I) \\
& =(c \wedge \neg e,(c \wedge \neg f \wedge d \wedge \neg e) I) .
\end{aligned}
$$

Hence,

$$
\begin{aligned}
(x, y I) *(p, q I) & =(x \wedge \neg p,(x \wedge \neg q \wedge y \wedge \neg p) I) \\
& \equiv(u, v I),
\end{aligned}
$$

where

$$
\begin{aligned}
u & =x \wedge \neg p=a \wedge \neg e \wedge(\neg c \vee e) \\
& =a \wedge \neg e \wedge \neg c=0, \\
v & =x \wedge \neg q \wedge y \wedge \neg p \\
& =a \wedge \neg e \wedge \neg f \wedge b \wedge \neg e \wedge(\neg c \vee e) \wedge \neg q \\
& =a \wedge \neg e \wedge \neg f \wedge b \wedge \neg e \wedge \neg c \wedge(\neg c \vee f \vee \neg d \vee e) \\
& =(a \wedge \neg c \wedge \neg e \wedge \neg f \wedge b) \vee(a \wedge \neg d \wedge b \wedge \neg c \wedge \neg e \wedge \neg f) \\
& =0 \vee 0=0 .
\end{aligned}
$$

This shows that $(u, v I)=(0,0)$ and so $((a, b I) *(e, f I)) *$ $((c, d I) *(e, f I))=(0,0)$. Similar computations show that $((e, f I) *(c, d I)) *((e, f I) *(a, b I))=(0,0)$.

(2) Put

$$
\text { LHS }=((a, b I) *(c, d I)) *(e, f I)=(x, y I) *(e, f I),
$$


where

$$
(x, y I)=(a, b I) *(c, d I)=(a \wedge \neg c,(a \wedge \neg d \wedge b \wedge \neg c) I) .
$$

Therefore,

$$
\begin{aligned}
(x, y I) *(e, f I) & =(x \wedge \neg e,(x \wedge \neg f \wedge y \wedge \neg e) I) \\
& \equiv(u, v I) .
\end{aligned}
$$

Now, we have

$$
\begin{aligned}
u & =x \wedge \neg e=a \wedge \neg c \wedge \neg e, \\
v & =x \wedge \neg f \wedge y \wedge \neg e=x \wedge \neg f \wedge y \wedge \neg e \\
& =a \wedge \neg c \wedge \neg f \wedge \neg e \wedge \neg d \wedge b .
\end{aligned}
$$

Thus,

$$
\text { LHS }=(a \wedge \neg c \wedge \neg e,(a \wedge \neg c \wedge \neg f \wedge \neg e \wedge \neg d \wedge b) I) .
$$

Similarly, it can be shown that

$$
\begin{aligned}
\text { RHS } & =((a, b I) *(e, f I)) *(c, d I) \\
& =(a \wedge \neg c \wedge \neg e,(a \wedge \neg c \wedge \neg f \wedge \neg e \wedge \neg d \wedge b) I) .
\end{aligned}
$$

(3) Put

$$
\begin{aligned}
\mathrm{LHS}= & ((a, b I) *(e, f I)) \\
& *((c, d I) *(e, f I)) *((a, b I) *(c, d I)) \\
\equiv & ((x, y I) *(p, q I)) *(u, v I),
\end{aligned}
$$

where

$$
\begin{aligned}
& (x, y I)=(a, b I) *(e, f I)=(a \wedge \neg c,(a \wedge \neg f \wedge b \wedge \neg e) I), \\
& (p, q I)=(c, d I) *(e, f I)=(c \wedge \neg e,(c \wedge \neg f \wedge d \wedge \neg e) I), \\
& (u, v I)=(a, b I) *(c, d I)=(a \wedge \neg c,(a \wedge \neg d \wedge b \wedge \neg c) I) .
\end{aligned}
$$

Thus, we have

$$
\begin{aligned}
(x, y I) *(p, q I) & =(x \wedge \neg p,(x \wedge \neg q \wedge y \wedge \neg p) I) \\
& \equiv(g, h I) .
\end{aligned}
$$

Now,

$$
\begin{aligned}
(g, h I) *(u, v I) & =(g \wedge \neg u,(g \wedge \neg v \wedge h \wedge \neg u) I) \\
& \equiv(m, k I),
\end{aligned}
$$

where

$$
\begin{aligned}
m & =g \wedge \neg u \\
& =x \wedge \neg p \wedge(\neg a \wedge c) \\
& =a \wedge \neg e \wedge(\neg c \vee e) \wedge(\neg a \vee c) \\
& =a \wedge \neg e \wedge \neg c \wedge(\neg a \vee c) \\
& =0 \vee 0=0, \\
k & =g \wedge \neg v \wedge h \wedge \neg u \\
& =x \wedge \neg p \wedge \neg v \wedge \neg q \wedge y \wedge(\neg a \vee c) \\
& =a \wedge \neg e \wedge(\neg c \vee e) \wedge(\neg a \vee c) \wedge y \wedge \neg v \wedge \neg q \\
& =a \wedge \neg e \wedge \neg c \wedge(\neg a \vee c) \wedge y \wedge \neg v \wedge \neg q \\
& =(0 \vee 0) \wedge y \wedge \neg v \wedge \neg q=0 .
\end{aligned}
$$

Since $(m, k I)=(0,0)$, it follows that LHS $=(0,0)$. Hence this completes the proof.

Theorem 9. Let $(X(I), *,(0,0))$ be a neutrosophic BCI/BCKalgebra. Then

(1) $X(I)$ is not commutative even if $X$ is commutative;

(2) $X(I)$ is not implicative even if $X$ is implicative.

Proof. (1) Suppose that $X$ is commutative. Let $(a, b I),(c, d I) \in$ $X(I)$. Then

$$
\begin{aligned}
& (a, b I) *((a, b I) *(c, d I)) \\
& =(a, b I) *(a * c,(a * d \wedge b * c \wedge b * d) I) \\
& =(a *(a * c),(a *(a * d \wedge b * c \wedge b * d) \wedge b *(a * c) \\
& \quad \wedge b *(a * d \wedge b * c \wedge b * d)) I) \\
& \equiv(u, v I),
\end{aligned}
$$

where

$$
\begin{aligned}
u= & a *(a * c)=c *(c * a), \\
v= & a *(a * d \wedge b * c \wedge b * d) \wedge b *(a * c) \\
& \wedge b *(a * d \wedge b * c \wedge b * d) \\
= & a *(a * d) \wedge a *(b * c) \wedge a *(b * d) \\
& \wedge b *(a * c) \wedge b *(a * d) \\
& \wedge b *(b * c) \wedge b *(b * d) \\
= & d *(d * a) \wedge a *(b * c) \wedge a *(b * d) \\
& \wedge b *(a * c) \wedge b *(a * d) \\
& \wedge c *(c * b) \wedge d *(d * b) .
\end{aligned}
$$


Also,

$$
\begin{aligned}
& (c, d I) *((c, d I) *(a, b I)) \\
& =(c, d I) *(c * a,(c * b \wedge d * a \wedge d * b) I) \\
& =(c *(c * a),(c *(c * b \wedge d * a \wedge d * b) \wedge d *(c * a) \\
& \quad \wedge d *(c * b \wedge d * a \wedge d * b)) I) \\
& \equiv(p, q I),
\end{aligned}
$$

where

$$
\begin{aligned}
p= & c *(c * a)=u, \\
q= & c *(c * b \wedge d * a \wedge d * b) \wedge d *(c * a) \\
& \wedge d *(c * b \wedge d * a \wedge d * b) \\
= & c *(c * b) \wedge c *(d * a) \wedge c *(d * b) \wedge d *(c * a) \\
& \wedge d *(c * b) \wedge d *(d * a) \wedge d *(d * b) \\
& \neq v .
\end{aligned}
$$

This shows that $(a, b I) *((a, b I) *(c, d I)) \neq(c, d I) *((c, d I) *$ $(a, b I))$ and therefore $X(I)$ is not commutative.

(2) Suppose that $X$ is implicative. Let $(a, b I),(c, d I) \in$ $X(I)$. Then

$$
\begin{aligned}
& (a, b I) *((c, d I) *(a, b I)) \\
& =(a, b I) *(c * a,(c * b \wedge d * a \wedge d * b) I) \\
& =(a *(c * a),(a *(c * b \wedge d * a \wedge d * b) \wedge b *(c * a) \\
& \quad \wedge b *(c * b \wedge d * a \wedge d * b)) I) \\
& \quad \equiv(u, v I),
\end{aligned}
$$

where

$$
\begin{aligned}
u= & a *(c * a)=a, \\
v= & a *(c * b \wedge d * a \wedge d * b) \wedge b *(c * a) \\
& \wedge b *(c * b \wedge d * a \wedge d * b) \\
= & a *(c * b) \wedge a *(d * a) \wedge a *(d * b) \wedge b *(c * a) \\
& \wedge b *(c * b) \wedge b *(d * a) \wedge b *(d * b) \\
\neq & b .
\end{aligned}
$$

Hence, $(a, b I) \neq(a, b I) *((c, d I) *(a, b I))$ and so $X(I)$ is not implicative.

Definition 10. Let $(X(I), *,(0,0))$ be a neutrosophic BCI/ BCK-algebra. A nonempty subset $A(I)$ is called a neutrosophic subalgebra of $X(I)$ if the following conditions hold:

(1) $(0,0) \in A(I)$;
(2) $(a, b I) *(c, d I) \in A(I)$ for all $(a, b I),(c, d I) \in A(I)$;

(3) $A(I)$ contains a proper subset which is a $\mathrm{BCI} / \mathrm{BCK}$ algebra.

If $A(I)$ does not contain a proper subset which is a $\mathrm{BCI} / \mathrm{BCK}$ algebra, then $A(I)$ is called a pseudo neutrosophic subalgebra of $X(I)$.

Example 11. Any neutrosophic subgroup of the commutative neutrosophic group $(X(I),+)$ of Example 2 is a neutrosophic BCI-subalgebra.

Theorem 12. Let $(X(I), *,(0,0))$ be a neutrosophic BCKalgebra and for $a \neq 0$ let $A_{(a, a I)}(I)$ be a subset of $X(I)$ defined by

$$
A_{(a, a I)}(I)=\{(x, y I) \in X(I):(x, y I) *(a, a I)=(0,0)\} .
$$

Then,

(1) $A_{(a, a I)}(I)$ is a neutrosophic subalgebra of $X(I)$;

(2) $A_{(a, a I)}(I) \subseteq A_{(0,0)}(I)$.

Proof. (1) Obviously, $(0,0) \in A_{(a, a I)}(I)$ and $A_{(a, a I)}(I)$ contains a proper subset which is a BCK-algebra. Let $(x, y I),(p, q I) \in$ $A_{(a, a I)}(I)$. Then $(x, y I) *(a, a I)=(0,0)$ and $(p, q I) *(a, a I)=$ $(0,0)$ from which we obtain $x * a=0, x * a \wedge y * a=0, p * a=0$, and $p * a \wedge q * a=0$. Since $a \neq 0$, we have $x=y=p=q=a$. Now,

$$
\begin{aligned}
&((x, y I) *(p, q I)) *(a, a I) \\
&=((x * p),(x * q \wedge y * p \wedge y * q) I) *(a, a I) \\
&=((x * p) * a,(((x * p) * a) \wedge(x * q \wedge y * p \\
&\quad \wedge y * q) * a) I) \\
&=((a * a) * a,((a * a) * a) I) \\
&=(0 * a,(0 * a) I) \\
&=(0,0) .
\end{aligned}
$$

This shows that $(x, y I) *(p, q I) \in A_{(a, a I)}(I)$ and the required result follows.

(2) Follows.

Definition 13. Let $(X(I), *,(0,0))$ and $\left(X^{\prime}(I), \circ,\left(0^{\prime}, 0^{\prime}\right)\right)$ be two neutrosophic BCI/BCK-algebras. A mapping $\phi$ : $X(I) \rightarrow X^{\prime}(I)$ is called a neutrosophic homomorphism if the following conditions hold:

(1) $\phi((a, b I) *(c, d I))=\phi((a, b I)) \circ \phi((c, d I)), \forall(a, b I)$, $(c, d I) \in X(I)$

(2) $\phi((0, I))=(0, I)$.

In addition,

(3) if $\phi$ is injective, then $\phi$ is called a neutrosophic monomorphism; 
(4) if $\phi$ is surjective, then $\phi$ is called a neutrosophic epimorphism;

(5) if $\phi$ is a bijection, then $\phi$ is called a neutrosophic isomorphism. A bijective neutrosophic homomorphism from $X(I)$ onto $X(I)$ is called a neutrosophic automorphism.

Definition 14. Let $\phi: X(I) \rightarrow Y(I)$ be a neutrosophic homomorphism of neutrosophic BCK/BCI-algebras. Consider the following:

(1) $\operatorname{Ker} \phi=\{(a, b I) \in X(I): \phi((a, b I))=(0,0)\}$;

(2) $\operatorname{Im} \phi=\{\phi((a, b I)) \in Y(I):(a, b I) \in X(I)\}$.

Example 15. Let $(X(I), *,(0,0))$ be a neutrosophic BCI/BCKalgebra and let $\phi: X(I) \rightarrow X(I)$ be a mapping defined by

$$
\phi((a, b I))=(a, b I) \quad \forall(a, b I) \in X(I) .
$$

Then $\phi$ is a neutrosophic isomorphism.

Lemma 16. Let $\phi: X(I) \rightarrow X^{\prime}(I)$ be a neutrosophic homomorphism from a neutrosophic BCI/BCK-algebra $X(I)$ into a neutrosophic BCI/BCK-algebra $X^{\prime}(I)$. Then $\phi((0,0))=$ $\left(0^{\prime}, 0^{\prime}\right)$.

Proof. It is straightforward.

Theorem 17. Let $\phi: X(I) \rightarrow Y(I)$ be a neutrosophic homomorphism of neutrosophic BCK/BCI-algebras. Then $\phi$ is a neutrosophic monomorphism if and only if $\operatorname{Ker} \phi=\{(0,0)\}$.

Proof. The proof is the same as the classical case and so is omitted.

Theorem 18. Let $X(I), Y(I)$, and $Z(I)$ be neutrosophic $B C I / B C K$-algebras. Let $\phi: X(I) \rightarrow Y(I)$ be a neutrosophic epimorphism and let $\psi: X(I) \rightarrow Z(I)$ be a neutrosophic homomorphism. If $\operatorname{Ker} \phi \subseteq \operatorname{Ker} \psi$, then there exists a unique neutrosophic homomorphism $v: Y(I) \rightarrow Z(I)$ such that $\nu \phi=\psi$. The following also hold:

(1) $\operatorname{Ker} v=\phi(\operatorname{Ker} \psi)$;

(2) $\operatorname{Im} v=\operatorname{Im} \psi$;

(3) $\nu$ is a neutrosophic monomorphism if and only if $\operatorname{Ker} \phi=\operatorname{Ker} \psi$;

(4) $v$ is a neutrosophic epimorphism if and only if $\psi$ is a neutrosophic epimorphism.

Proof. The proof is similar to the classical case and so is omitted.

Theorem 19. Let $X(I), Y(I), Z(I)$ be neutrosophic BCI/BCKalgebras. Let $\phi: X(I) \rightarrow Z(I)$ be a neutrosophic homomorphism and let $\psi: Y(I) \rightarrow Z(I)$ be a neutrosophic monomorphism such that $\operatorname{Im} \phi \subseteq \operatorname{Im} \psi$. Then there exists a unique neutrosophic homomorphism $\mu: X(I) \rightarrow Y(I)$ such that $\phi=\psi \mu$. Also,

(1) $\operatorname{Ker} \mu=\operatorname{Ker} \phi$;
(2) $\operatorname{Im} \mu=\psi^{-1}(\operatorname{Im} \phi)$;

(3) $\mu$ is a neutrosophic monomorphism if and only if $\phi$ is a neutrosophic monomorphism;

(4) $\mu$ is a neutrosophic epimorphism if and only if $\operatorname{Im} \psi=$ $\operatorname{Im} \phi$.

Proof. The proof is similar to the classical case and so is omitted.

\section{Conflict of Interests}

The authors declare that there is no conflict of interests regarding the publication of this paper.

\section{References}

[1] Y. Imai and K. Iséki, "On axiom systems of propositional calculi, XIV," Proceedings of the Japan Academy, vol. 42, pp. 19-22, 1966.

[2] K. Iséki, "An algebra related with a propositional calculus," Proceedings of the Japan Academy, vol. 42, pp. 26-29, 1966.

[3] Y. S. Huang, BCI-Algebra, Science Press, Beijing, China, 2006.

[4] K. Iséki, "On BCI-algebras," Kobe University. Mathematics Seminar Notes, vol. 8, no. 1, pp. 125-130, 1980.

[5] K. Iséki and S. Tanaka, "An introduction to the theory of BCKalgebras," Mathematica Japonica, vol. 23, no. 1, pp. 1-26, 1978.

[6] J. M. Jie and Y. B. Jun, BCK-Algebras, Kyung Moon Sa Co., Seoul, Republic of Korea, 1994.

[7] C. C. Chang, "Algebraic analysis of many valued logics," Transactions of the American Mathematical Society, vol. 88, no. 2, pp. 467-490, 1958.

[8] F. Smarandache, A Unifying Field in Logics: Neutrosophic Logic, Neutrosophy, Neutrosophic Set, Neutrosophic Probability, American Research Press, Rehoboth, NM, USA, 2003.

[9] F. Smarandache, Introduction to Neutrosophic Statistics, Sitech and Education Publishing, Craiova, Romania, 2014.

[10] F. Smarandache, Neutrosophy/Neutrosophic Probability, Set, and Logic, American Research Press, Rehoboth, Mass, USA, 1998.

[11] W. B. V. Kandasamy and F. Smarandache, Some Neutrosophic Algebraic Structures and Neutrosophic N-Algebraic Structures, Hexis, Phoenix, Ariz, USA, 2006.

[12] W. B. Vasantha Kandasamy and F. Smarandache, Neutrosophic Rings, Hexis, Phoenix, Ariz, USA, 2006.

[13] A. A. A. Agboola, A. D. Akinola, and O. Y. Oyebola, "Neutrosophic rings I," International Journal of Mathematical Combinatorics, vol. 4, pp. 1-14, 2011.

[14] A. A. A. Agboola, E. O. Adeleke, and S. A. Akinleye, "Neutrosophic rings II," International Journal of Mathematical Combinatorics, vol. 2, pp. 1-8, 2012.

[15] A. A. A. Agboola, A. O. Akwu, and Y. T. Oyebo, "Neutrosophic groups and neutrosopic subgroups," International Journal of Mathematical Combinatorics, vol. 3, pp. 1-9, 2012.

[16] A. A. Agboola and B. Davvaz, "Introduction to neutrosophic hypergroups," ROMAI Journal, vol. 9, no. 2, pp. 1-10, 2013.

[17] A. A. A. Agboola and B. Davvaz, "On neutrosophic canonical hypergroups and neutrosophic hyperrings," Neutrosophic Sets and Systems, vol. 2, pp. 34-41, 2014. 


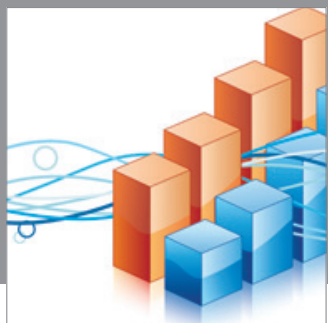

Advances in

Operations Research

mansans

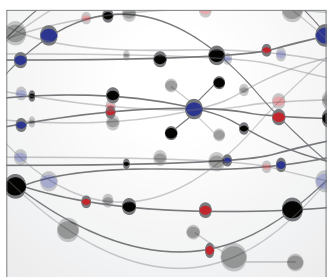

The Scientific World Journal
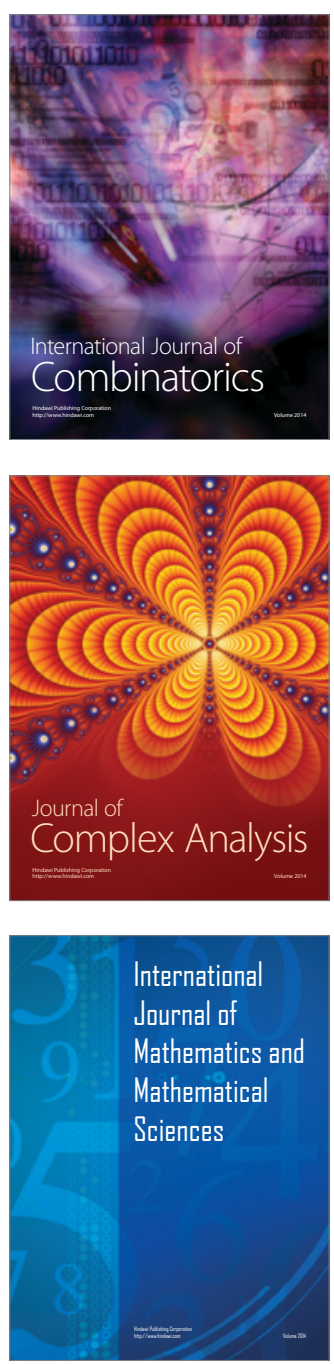
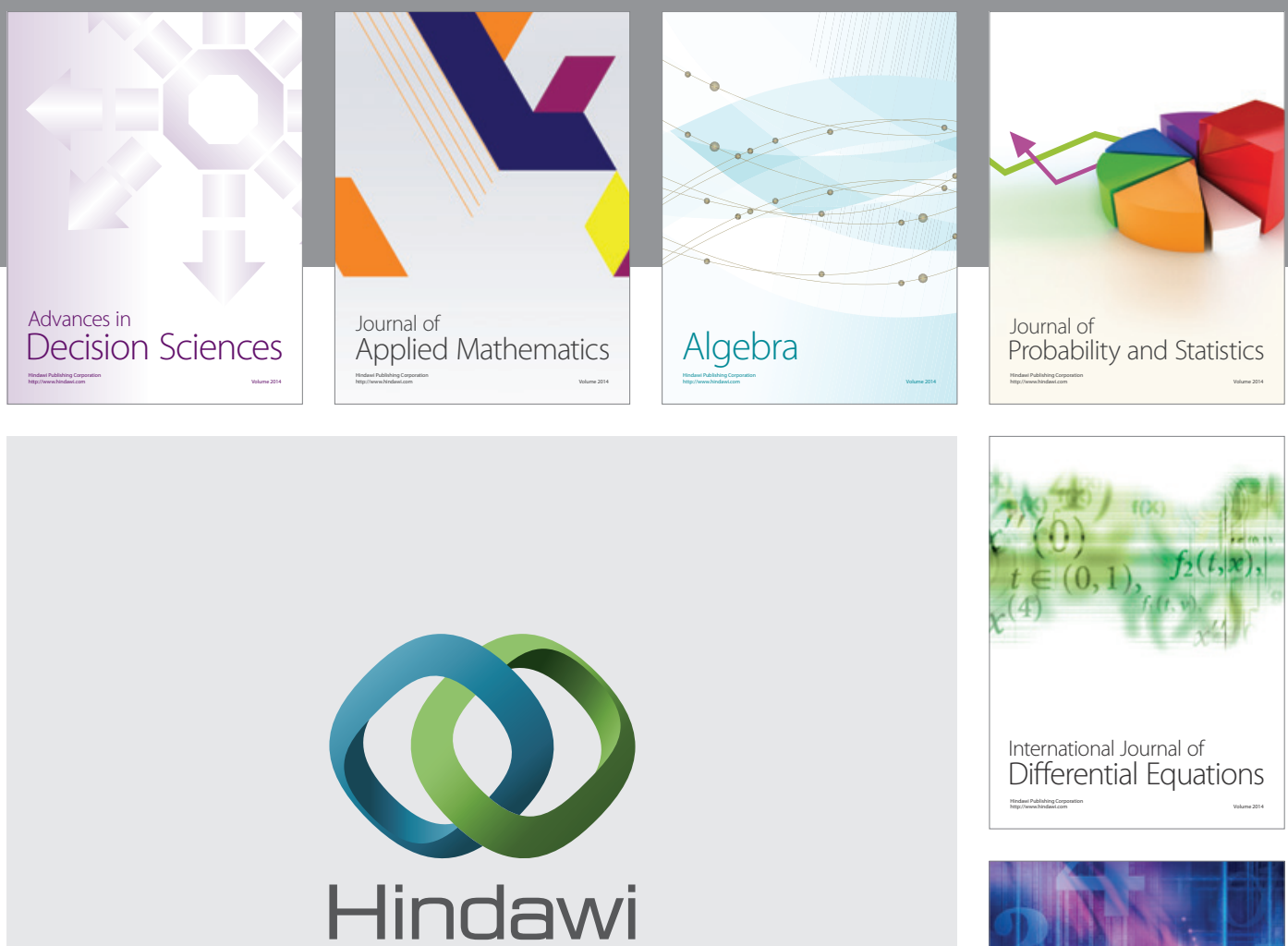

Submit your manuscripts at http://www.hindawi.com
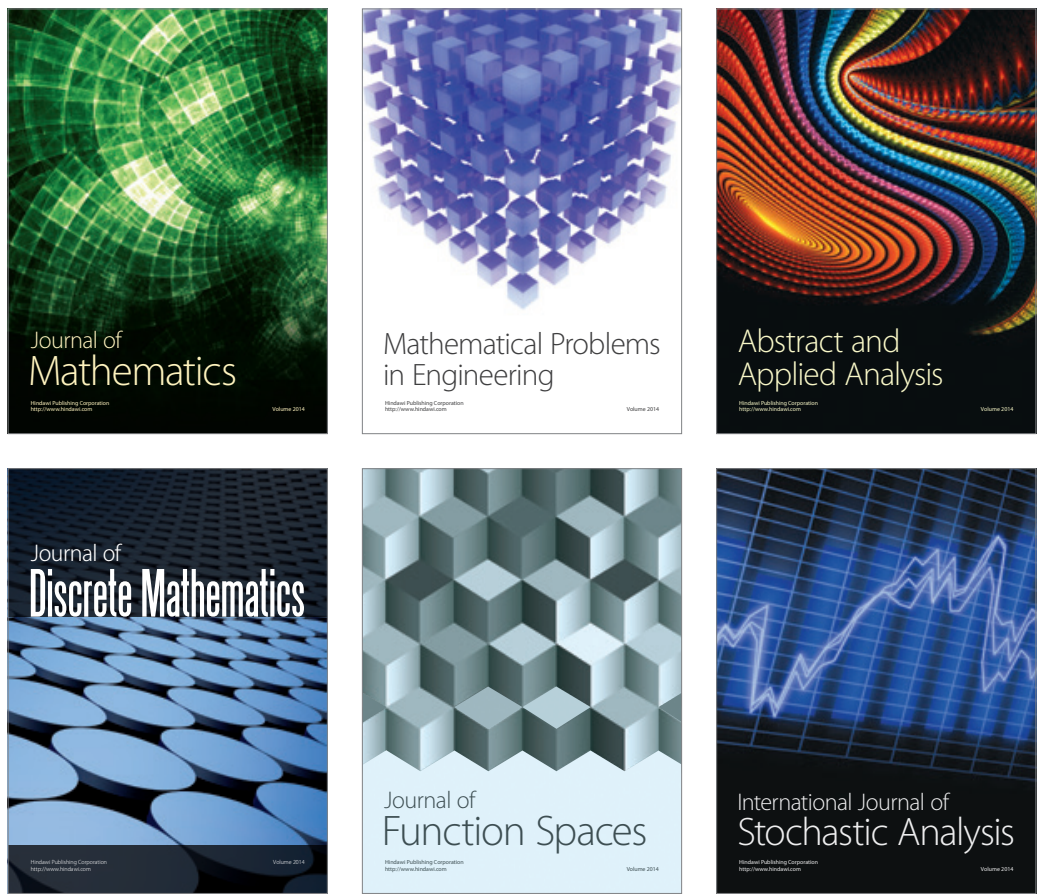

Journal of

Function Spaces

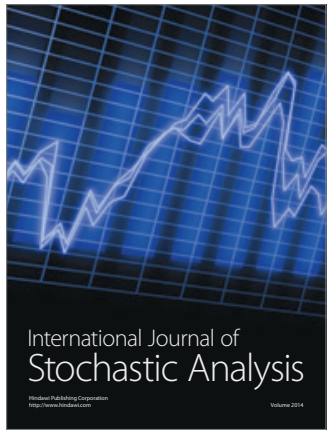

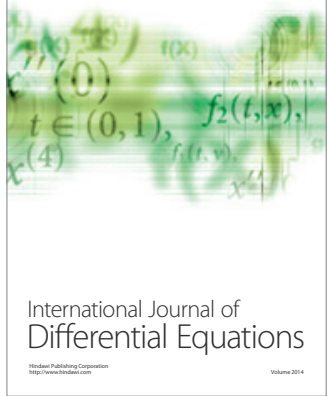
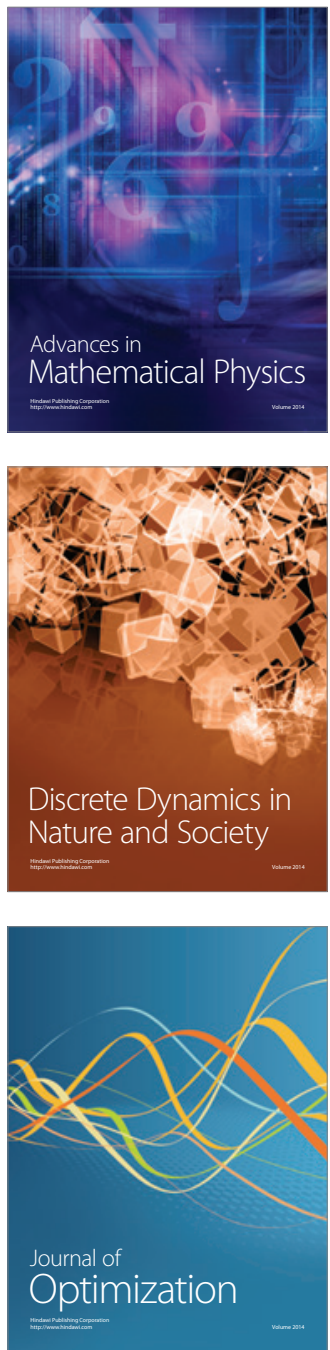